\title{
Uniqueness Properties of The Solution of The Inverse Problem for The Sturm-Liouville Equation With Discontinuous Leading Coefficient
}

\author{
ANAR ADILOGLU ${ }^{1}$, MEHMET GÜRDAL ${ }^{2}$ and AYŞE N. KINCI ${ }^{2}$ \\ ${ }^{1}$ Department of Mathematics, Faculty of Science, Cumhuriyet University, 58140 Sivas, Turkey \\ ${ }^{2}$ Department of Mathematics, Suleyman Demirel University, 32260, Isparta, Turkey
}

Manuscript received on February 5, 2016; accepted for publication on January 27, 2017

\begin{abstract}
The present paper studies uniqueness properties of the solution of the inverse problem for the SturmLiouville equation with discontinuous leading coefficient and the separated boundary conditions. It is proved that the considered boundary-value is uniquely reconstructed, i.e. the potential function of the equation and the constants in the boundary conditions are uniquely determined by given Weyl function or by the given spectral data.
\end{abstract}

Key words: Asymptotic formulas for eigenvalues, boundary value problems, inverse problems, spectral analysis of ordinary differential operators, Sturm-Liouville theory, transformation operator.

\section{INTRODUCTION}

This paper is concerned with the uniqueness theorems for the solution of some inverse spectral problems for the boundary value problem

$$
\begin{gathered}
-y^{\prime \prime}+q(x) y=\lambda^{2} \rho(x) y, 0 \leq x \leq \pi \\
y^{\prime}(0)-h y(0)=0, y^{\prime}(\pi)+h_{1} y(\pi)=0,
\end{gathered}
$$

where $q(x)$ is real-valued function in $L_{2}(0, \pi), \lambda$ is a complex parameter, $h, h_{1}$ are real numbers,

$$
\rho(x)= \begin{cases}1 & , 0 \leq x \leq a \\ \alpha^{2} & , a<x<\pi\end{cases}
$$

Correspondence to: Mehmet Gürdal

E-mail: gurdalmehmet@sdu.edu.tr 
with $a \in(0, \pi), \alpha \neq 1$.

Inverse spectral problems consist in recovering differential operators from their spectral characteristics (see Marchenko 2011, Levitan 1987). Such problems arise in many areas of science and engineering (see Hald 1980, Krueger 1982, Willis 1984). The goal of this work is to prove the uniqueness theorems for the solution of the inverse problem which determines the potential function $q(x)$ and the constants $h, h_{1}$ by the Weyl function or by the spectral data of the boundary value problem $(1)-(2)$.

In the classical case $\rho(x)=1$, the direct and inverse problems for the Sturm-Liouville operators have been completely studied (see Marchenko 2011, Levitan 1987, Levitan and Gasymov 1964, Freiling and Yurko 2001, Hryniv and Mykytyuk 2003, 2004, and the references therein). Direct and inverse problems for the discontinuous Sturm-Liouville boundary-value problems in different settings have been studied in Hald (1984), Andersson (1988), Guseinov and Pashaev (2002), Carlson (1994), Yurko (2000), Gasymov (1977), Amirov (2006), Mamedov (2006, 2010), Mamedov and Palamut (2009)) and other works. Note that, the direct and inverse spectral problem for the equation (1) with simple boundary conditions on the interval $(0, \pi)$ recently has been investigated in Akhmedova and Huseynov (2010) by using a new integral representations of the special solutions of Eq. (1).

The spectral analysis of the boundary value problem (1) - (2) was examined in Adiloglu and Amirov (2013) where useful integral representations for two linearly independent solutions of equation (1) were constructed (see also Akhmedova and Huseynov 2010), the asymptotic formulas for the eigenvalues and eigenfunctions were obtained, completeness and expansion theorems for the system of the eigenfunctions were proved. Using the results of Adiloglu and Amirov (2013), in the present paper, we investigate uniqueness properties of the solution of the inverse problems for the problem (1) - (2) and study some other types boundary value problems related with the equation (1). In section 2 we prove that the boundary-value problem (1) - (2) is uniquely reconstructed, i.e. the potential function $q(x)$ and the constants $h, h_{1}$ are uniquely determined by given Weyl function or by the given spectral data. We also show that in the special case the potential $q(x)$ and the coefficient $h$ can be determined by the one spectrum only. In section 3 we investigate the properties of the spectral characteristics of two boundary-value problems related to Eq. (1).

\section{UNIQUENESS OF THE SOLUTION OF THE INVERSE PROBLEM}

Let $s(x, \lambda), c(x, \lambda)$ be solutions of Eq. (1) with initial conditions

$$
s(0, \lambda)=c^{\prime}(0, \lambda)=0, s^{\prime}(0, \lambda)=c(0, \lambda)=1
$$

and $\varphi(x, \lambda), \psi(x, \lambda)$ be solutions of (1) under conditions at $\pi$ :

$$
\varphi(\pi, \lambda)=\psi^{\prime}(\pi, \lambda)=-1, \varphi^{\prime}(\pi, \lambda)=\psi(\pi, \lambda)=0 .
$$

Then $w_{1}(x, \lambda)=h s(x, \lambda)+c(x, \lambda)$ and $w_{2}(x, \lambda)=\psi(x, \lambda)-h_{1} \varphi(x, \lambda)$ are solutions of $(1)$ with initial conditions

$$
w_{1}(0, \lambda)=1, w_{1}^{\prime}(0, \lambda)=h
$$

and

$$
w_{2}(\pi, \lambda)=-1, w_{2}^{\prime}(\pi, \lambda)=h_{1}
$$

respectively (see Adiloglu and Amirov 2013). 
Denote by $\Phi(x, \lambda)$ the solution of equation (1) satisfying conditions

$$
\Phi^{\prime}(0, \lambda)-h \Phi(0, \lambda)=1, \Phi^{\prime}(\pi, \lambda)+h_{1} \Phi(\pi, \lambda)=0 .
$$

We set $M(\lambda)=\Phi(0, \lambda)$ and consider the linearly independent solutions $s(x, \lambda)$ and $w_{1}(x, \lambda)$ of equation (1). We have

$$
\Phi(x, \lambda)=s(x, \lambda)-\frac{\triangle^{(0)}(\lambda)}{\triangle(\lambda)} w_{1}(x, \lambda)
$$

where

$$
\begin{gathered}
\triangle^{(0)}(\lambda)=s^{\prime}(\pi, \lambda)+h_{1} s(\pi, \lambda), \\
\triangle(\lambda)=w_{1}^{\prime}(\pi, \lambda)+h_{1} w_{1}(\pi, \lambda)=-w_{2}^{\prime}(0, \lambda)+h w_{2}(0, \lambda) .
\end{gathered}
$$

Therefore,

$$
M(\lambda)=\Phi(0, \lambda)=-\frac{\triangle^{(0)}(\lambda)}{\triangle(\lambda)}
$$

Then Eq. (4) can be written as

$$
\Phi(x, \lambda)=s(x, \lambda)+M(\lambda) w_{1}(x, \lambda)
$$

Additionally we see that the solution $w_{2}(x, \lambda)$ also satisfies the second one of the conditions (3). Consequently we obtain

$$
\Phi(x, \lambda)=-\frac{w_{2}(x, \lambda)}{\triangle(\lambda)} .
$$

We also have

$$
\left\langle w_{1}(x, \lambda), \Phi(x, \lambda)\right\rangle:=w_{1}(x, \lambda) \Phi^{\prime}(x, \lambda)-w_{1}^{\prime}(x, \lambda) \Phi(x, \lambda) \equiv 1 .
$$

The functions $\Phi(x, \lambda)$ and $M(\lambda)$ are called the Weyl solution and the Weyl function of the boundary value problem (1) - (2), respectively. From equation (5) we have that the Weyl function $M(\lambda)$ is a meromorphic function with simple poles at the points $\lambda=\lambda_{n}, n \geq 0$, where $\left\{\lambda_{n}^{2}\right\}$ are eigenvalues of the boundary value problem (1) - (2) (see Adiloglu and Amirov 2013).

We recall that the normalized numbers (see Adiloglu and Amirov 2013) $\alpha_{n}$ of the boundary value problem (1) - (2) are defined as

$$
\alpha_{n}=\int_{0}^{\pi} \rho(x) w_{1}^{2}\left(x, \lambda_{n}\right) d x, \quad(n=0,1,2, \ldots),
$$

the set $\left\{\lambda_{n}^{2}, \alpha_{n}\right\}_{n \geq 0}$ is the spectral data of the problem (1)-(2). Note that there exists the sequence $\beta_{n}$ such that

$$
\beta_{n} \alpha_{n}=\frac{\dot{\Delta}\left(\lambda_{n}\right)}{2 \lambda_{n}}
$$

(see Adiloglu and Amirov 2013).

The following theorem shows that the given spectral data uniquely determines the boundary- value problem $(1)-(2)$.

Theorem 1. The following formula holds $M(\lambda)=\sum_{n=0}^{\infty} \frac{1}{\alpha_{n}\left(\lambda-\lambda_{n}\right)}$. 
Proof. Since $\triangle^{(0)}(\lambda)=s^{\prime}(\pi, \lambda)+h_{1} s(\pi, \lambda)=w_{2}(0, \lambda)$ it follows from (Adiloglu and Amirov (2013)) (see formula (50), Adiloglu and Amirov 2013) that $\left|\triangle^{(0)}(\lambda)\right| \leq C e^{|\operatorname{Im} \lambda| \mu^{+}(\pi)}$, where $\mu^{+}(\pi)=\alpha \pi-\alpha a+a$. Since $|\triangle(\lambda)| \geq \widetilde{C_{\delta}}|\lambda| e^{|\operatorname{Im} \lambda| \mu^{+}(\pi)}, \lambda \in G_{\delta},|\lambda|>\rho_{0}>0$, where, $G_{\delta}=\left\{\lambda:\left|\lambda-\lambda_{n}^{0}\right| \geq \delta\right\}$ and $\widetilde{C_{\delta}}>0$ for some $\delta>0$, we have

$$
|M(\lambda)| \leq \frac{C_{\delta}}{|\lambda|}, \lambda \in G_{\delta},|\lambda| \geq \rho_{0}
$$

Further using the Lemma 1 (Adiloglu and Amirov (2013)) (see also the formula (44) there) we find

$$
\operatorname{Res}_{\lambda=\lambda_{n}} M(\lambda)=-\frac{\triangle^{(0)}\left(\lambda_{n}\right)}{\triangle\left(\lambda_{n}\right)}=-\frac{\beta_{n}}{\triangle\left(\lambda_{n}\right)}=\frac{1}{\alpha_{n}} .
$$

Let

$$
\begin{gathered}
I_{N}(\lambda)=\frac{1}{2 \pi i} \int_{\Gamma_{N}} \frac{M(p)}{\lambda-p} d p \\
\lambda \in D_{N}:=\left\{\lambda:|\lambda|<\left(N+\frac{1}{2}\right)^{2}\right\}, \Gamma_{N}=\partial D_{N} .
\end{gathered}
$$

Then by virtue of $(9)$ we have $\lim _{N \rightarrow \infty} I_{N}(\lambda)=0$. On the other hand by the residue theorem

$$
I_{N}(\lambda)=-M(\lambda)+\sum_{n=0}^{N} \frac{1}{\alpha_{n}\left(\lambda-\lambda_{n}\right)}
$$

which gives the desired results as $N \rightarrow+\infty$. Theorem is proved.

Now let the Weyl function $M(\lambda)$ of the boundary value problem (1) - (2) is given. In the following theorem we prove that the boundary problem $(1)-(2)$ is uniquely reconstructed, i.e. the potential function $q(x)$ and the constants $h, h_{1}$ are uniquely determined by given Weyl function.

Let us denote the boundary value problem (1) - (2) by $L=L\left(q(x), h, h_{1}\right)$ and the similar boundary value problem with the potential $\widetilde{q}(x)$ and boundary constants $\widetilde{h}, \widetilde{h}_{1}$ by $\widetilde{L}=L\left(\widetilde{q}(x), \widetilde{h}, \widetilde{h}_{1}\right)$. Then the following theorem is satisfied.

Theorem 2. Let the Weyl functions of the boundary value problem $L$ and $\widetilde{L}$ are $M(\lambda)$ and $\widetilde{M}(\lambda)$ respectively. If $M(\lambda)=\widetilde{M}(\lambda)$ then $L=\widetilde{L}$.

Proof. Define the matrix $P(x, \lambda)=\left[P_{i k}(x, \lambda)\right]_{i, k=1,2}$ by the formula

$$
P(x, \lambda)\left[\begin{array}{cc}
\widetilde{w}_{1}(x, \lambda) & \widetilde{\Phi}(x, \lambda) \\
\widetilde{w}_{1}^{\prime}(x, \lambda) & \widetilde{\Phi}^{\prime}(x, \lambda)
\end{array}\right]=\left[\begin{array}{cc}
w_{1}(x, \lambda) & \Phi(x, \lambda) \\
w_{1}^{\prime}(x, \lambda) & \Phi^{\prime}(x, \lambda)
\end{array}\right]
$$

where $\widetilde{w}_{1}(x, \lambda)$ and $\widetilde{\Phi}(x, \lambda)$ are solutions of the equation of the boundary value problem $\widetilde{L}$ which is identical to the solutions $w_{1}(x, \lambda)$ and $\Phi(x, \lambda)$. Since $\left\langle\widetilde{w}_{1}(x, \lambda), \widetilde{\Phi}(x, \lambda)\right\rangle \equiv 1$ we have

$$
\left\{\begin{array}{l}
w_{1}(x, \lambda)=P_{11}(x, \lambda) \widetilde{w}_{1}(x, \lambda)+P_{12}(x, \lambda) \widetilde{w}_{1}^{\prime}(x, \lambda) \\
\Phi(x, \lambda)=P_{11}(x, \lambda) \widetilde{\Phi}(x, \lambda)+P_{12}(x, \lambda) \widetilde{\Phi}^{\prime}(x, \lambda)
\end{array}\right.
$$


or

$$
\left\{\begin{array}{l}
P_{11}(x, \lambda)=w_{1}(x, \lambda) \widetilde{\Phi}^{\prime}(x, \lambda)-\widetilde{w}_{1}^{\prime}(x, \lambda) \Phi(x, \lambda) \\
P_{12}(x, \lambda)=\widetilde{w}_{1}(x, \lambda) \Phi(x, \lambda)-w_{1}(x, \lambda) \widetilde{\Phi}(x, \lambda)
\end{array}\right.
$$

Using the formula (7) in equation (13) we find

$$
\begin{aligned}
P_{11}(x, \lambda) & =1+\frac{1}{\triangle(\lambda)}\left\{w_{2}(x, \lambda)\left[\widetilde{w}_{1}^{\prime}(x, \lambda)-w_{1}^{\prime}(x, \lambda)\right]-\right. \\
& \left.=-w_{1}(x, \lambda)\left[\widetilde{w}_{2}^{\prime}(x, \lambda)-w_{2}^{\prime}(x, \lambda)\right]\right\} \\
P_{12}(x, \lambda) & =\frac{1}{\triangle(\lambda)}\left\{w_{1}(x, \lambda) \widetilde{w}_{2}(x, \lambda)-\widetilde{w}_{1}(x, \lambda) w_{2}(x, \lambda)\right\}
\end{aligned}
$$

Now using the estimations (49), (50) and (84) in (Adiloglu and Amirov (2013)) we have

$$
\left|P_{11}(x, \lambda)-1\right| \leq \frac{c_{\delta}}{|\lambda|},\left|P_{12}(x, \lambda)\right| \leq \frac{c_{\delta}}{|\lambda|}, \lambda \in G_{\delta},|\lambda| \geq \rho_{0}
$$

On the other hand from the equation (6) we obtain

$$
\begin{aligned}
P_{11}(x, \lambda) & =w_{1}(x, \lambda) \widetilde{s}^{\prime}(x, \lambda)-\widetilde{w}_{1}^{\prime}(x, \lambda) s(x, \lambda)+ \\
& +[\widetilde{M}(\lambda)-M(\lambda)] w_{1}(x, \lambda) \widetilde{w}_{1}^{\prime}(x, \lambda) \\
P_{12}(x, \lambda) & =\widetilde{w}_{1}(x, \lambda) s(x, \lambda)-w_{1}(x, \lambda) \widetilde{s}(x, \lambda)+ \\
& +[M(\lambda)-\widetilde{M}(\lambda)] w_{1}(x, \lambda) \widetilde{w}_{1}(x, \lambda)
\end{aligned}
$$

Thus if $M(\lambda)=\widetilde{M}(\lambda)$ then the functions $P_{11}(x, \lambda)$ and $P_{12}(x, \lambda)$ are entire in $\lambda$. Together with (16) this gives $P_{11}(x, \lambda) \equiv 1, P_{12}(x, \lambda) \equiv 0$. Then from $(12)$ we obtain $w_{1}(x, \lambda)=\widetilde{w}_{1}(x, \lambda), \Phi(x, \lambda)=\widetilde{\Phi}(x, \lambda)$ for all $x$ and $\lambda$. Since $w_{1}(x, \lambda)$ and $\widetilde{w}_{1}(x, \lambda)$ satisfy the equations

$$
-w_{1}^{\prime \prime}+q(x) w_{1}=\lambda^{2} \rho(x) w_{1} \text { and } \widetilde{w}_{1}^{\prime \prime}+\widetilde{q}(x) \widetilde{w}_{1}=\lambda^{2} \rho(x) \widetilde{w}_{1}
$$

correspondingly, substructing these equations we have

$$
(q(x)-\widetilde{q}(x)) w_{1}(x, \lambda)=0 .
$$

Therefore $q(x)=\widetilde{q}(x)$ a.e. on $(0, \pi)$. Further, because of $\Phi(x, \lambda)=\widetilde{\Phi}(x, \lambda)$ for all $x$ and $\lambda$ from the conditions (3) we also have that $h=\widetilde{h}, h_{1}=\widetilde{h_{1}}$. Consequently $L=\widetilde{L}$. Theorem is proved.

Theorem 3. Let $S=\left\{\lambda_{n}^{2}, \alpha_{n}\right\}_{n \geq 0}$ and $\widetilde{S}=\left\{\widetilde{\lambda}_{n}^{2}, \widetilde{\alpha}_{n}\right\}_{n \geq 0}$ are the spectral data of the problems $L$ and $\widetilde{L}$ respectively. If $\lambda_{n}=\widetilde{\lambda}_{n}, \alpha_{n}=\widetilde{\alpha}_{n}, n \geq 0$ then $L=\widetilde{L}$.

Proof. It is known (Adiloglu and Amirov (2013)) that the solutions $c(x, \lambda)$ and $s(x, \lambda)$ of equation (1) satisfying the conditions

$$
c(0, \lambda)=s^{\prime}(0, \lambda)=1, c^{\prime}(0, \lambda)=s(0, \lambda)=1
$$


are expressed as

$$
\begin{aligned}
& c(x, \lambda)=c_{0}(x, \lambda)+\int_{0}^{\mu^{+}(x)} N_{+}(x, t) \cos \lambda t d t \\
& s(x, \lambda)=s_{0}(x, \lambda)+\int_{0}^{\mu^{+}(x)} N_{-}(x, t) \frac{\sin \lambda t}{\lambda} d t
\end{aligned}
$$

respectively, where

$$
\begin{gathered}
c_{0}(x, \lambda)=r^{+}(x) \cos \lambda \mu^{+}(x)+r^{-}(x) \cos \lambda \mu^{-}(x) \\
s_{0}(x, \lambda)=r^{+}(x) \frac{\sin \lambda \mu^{+}(x)}{\lambda}+r^{-}(x) \frac{\sin \lambda \mu^{-}(x)}{\lambda} \\
r^{ \pm}(x)=\frac{1}{2}\left(1 \pm \frac{1}{\sqrt{\rho(x)}}\right), \mu^{ \pm}(x)= \pm x \sqrt{\rho(x)}+a(1 \pm \sqrt{\rho(x)})
\end{gathered}
$$

and $N_{ \pm}(x, \cdot) \in L_{1}\left(0, \mu^{+}(x)\right)$ for each $x \in[0, \pi]$. Using (17) and (18) we have

$$
w_{1}(x, \lambda)=w_{1}^{(0)}(x, \lambda)+\int_{0}^{\mu^{+}(x)} W_{1}(x, t) \cos \lambda t d t
$$

where

$$
\begin{gathered}
w_{1}^{(0)}(x, \lambda)=c_{0}(x, \lambda)+h s_{0}(x, \lambda) \\
W_{1}(x, t)=N_{+}(x, t)+h \int_{t}^{\mu^{+}(x)} N_{-}(x, \xi) d \xi .
\end{gathered}
$$

Clearly $W_{1}(x, \cdot) \in L_{1}\left(0 ; \mu^{+}(x)\right)$ for all $x \in[0, \pi]$. Note that the function $W_{1}(x, t)$ also satisfies the conditions (see Adiloglu and Amirov 2013)

$$
\begin{gathered}
\frac{d}{d x} W_{1}\left(x, \mu^{+}(x)\right)=\frac{1}{4 \sqrt{\rho(x)}}\left(1+\frac{1}{\sqrt{\rho(x)}}\right) q(x), \\
\frac{d}{d x}\left\{W_{1}\left(x, \mu^{-}(x)+0\right)-W_{1}\left(x, \mu^{-}(x)-0\right)\right\}=\frac{1}{4 \sqrt{\rho(x)}}\left(1-\frac{1}{\sqrt{\rho(x)}}\right) q(x) .
\end{gathered}
$$

Since

$$
s_{0}(x, \lambda)=\int_{0}^{\mu^{+}(x)} r(x, t) \cos \lambda t d t
$$

where $r(x, t)=\left\{\begin{array}{ll}1 & , 0<t<\mu^{-}(x) \\ \alpha^{+} & , \mu^{-}(x)<t<\mu^{+}(x)\end{array}\right.$ the equation (19) can be written as

$$
w_{1}(x, \lambda)=c_{0}(x, \lambda)+\int_{0}^{\mu^{+}(x)} W(x, t) \cos \lambda t d t
$$


where

$$
W(x, t)=W_{1}(x, t)+h r(x, t) .
$$

It is clear that if $0 \leq x \leq a$ then

$$
w_{1}(x, \lambda)=\cos \lambda x+\int_{0}^{x} W(x, t) \cos \lambda t d t
$$

where $W(x, t)=W_{1}(x, t)+h$ is continuous kernel. Then we can see the equation (26) as a Volterra integral equation with respect to $\cos \lambda x$. From the theory of the Volterra integral equations, we know that the equation (26) is then uniquely solvable and the solution is

$$
\cos \lambda x=w_{1}(x, \lambda)+\int_{0}^{x} \widehat{W}(x, t) w_{1}(t, \lambda) d t
$$

where $\widehat{W_{1}}(x, t)$ is a continuous kernel.

Let now $x>a$. In this case the equation (24) is written as

$$
w_{1}(x, \lambda)=\alpha^{+} \cos \lambda \mu^{+}(x)+\alpha^{-} \cos \lambda\left(2 a-\mu^{+}(x)\right)++\int_{0}^{\mu^{+}(x)} W(x, t) \cos \lambda t d t
$$

where $\alpha^{ \pm}=\frac{1}{2}\left(1 \pm \frac{1}{\alpha}\right)$. Here the kernel $W(x, t)$ has a jump discontinuity at $t=\mu^{-}(x)$. Clearly, $\mu^{+}(x)>a$ and $0<\mu^{-}(x)<a$ when $x>a$ and therefore (28) takes the form

$$
w_{1}(x, \lambda)=\alpha^{+} \cos \lambda \mu^{+}(x)+\int_{a}^{\mu^{+}(x)} W(x, t) \cos \lambda t d t+h(x, \lambda),
$$

where

$$
h(x, \lambda)=\alpha^{-} \cos \lambda \mu^{-}(x)+\int_{0}^{a} W(x, t) \cos \lambda t d t
$$

Now using (27) we obtain that

$$
\begin{aligned}
h(x, \lambda) & =\alpha^{-} w_{1}\left(\mu^{-}(x), \lambda\right)+\alpha^{-} \int_{0}^{\mu^{-}(x)} \widehat{W}\left(\mu^{-}(x), t\right) w_{1}(t, \lambda) d t \\
-\alpha^{-} \int_{0}^{\mu^{-}(x)}\left(\int_{a}^{x} U\left(x, \mu^{+}(s)\right) \widehat{W}\left(\mu^{-}(s), t\right) d s\right) w_{1}(t, \lambda) d t & \\
& +\int_{0}^{a}\left(W(x, t)+\int_{t}^{a} W(x, s) \widehat{W}(s, t) d s\right) w_{1}(t, \lambda) d t,
\end{aligned}
$$


where $U\left(x, \mu^{+}(t)\right)$ is a continuous kernel. Therefore the equation (29) is written as

$$
w_{1}(x, \lambda)-h(x, \lambda)=\alpha^{+} \cos \lambda \mu^{+}(x)+\alpha^{+} \int_{a}^{x} W\left(x, \mu^{+}(t)\right) \cos \lambda \mu^{+}(x) d t,
$$

where the kernel $W\left(x, \mu^{+}(t)\right)$ is continuous. Hence, we obtain the Volterra integral equation

$$
w_{1}(x, \lambda)-h(x, \lambda)=(I+W) \alpha^{+} \cos \lambda \mu^{+}(x),
$$

where

$$
(I+W) f(x)=f(x)+\int_{a}^{x} W\left(x, \mu^{+}(t)\right) f(t) d t
$$

Solving the Volterra integral equation (33) with respect to $\alpha^{+} \cos \lambda \mu^{+}(x)$, we find

$$
\alpha^{+} \cos \lambda \mu^{+}(x)=w_{1}(x, \lambda)-h(x, \lambda)+\int_{a}^{x} U\left(x, \mu^{+}(t)\right)\left[w_{1}(t, \lambda)-h(t, \lambda)\right] d t .
$$

Taking into account, the expression for $h(x, \lambda)$, we have

$$
\cos \lambda \mu^{+}(x)=\frac{1}{\alpha^{+}} w_{1}(x, \lambda)-\frac{\alpha^{-}}{\alpha^{+}} w_{1}\left(\mu^{-}(x), \lambda\right)+\int_{0}^{x} \widehat{W}(x, t) w_{1}(t, \lambda) d t
$$

where $\widehat{W}(x, t)$ is a kernel with jump at $t=\mu^{-}(x)$. Consequently, we have

$$
w_{1}(x, \lambda)=\widetilde{w_{1}}(x, \lambda)+\int_{0}^{x} H(x, t) \widetilde{w_{1}}(t, \lambda) d t,
$$

where $H(x, t)$ is a kernel with jump at $t=\mu^{-}(x)$.

Let $f(x) \in L_{2}(0, \pi)$. (37) implies that

$$
\int_{0}^{\pi} \rho(x) f(x) w_{1}(x, \lambda) d x=\int_{0}^{\pi} \rho(x) g(x) \widetilde{w_{1}}(x, \lambda) d x
$$

where $g(x)=f(x)+\int_{x}^{\pi} H(t, x) f(t) d t$. Hence for all $n \geq 0$

$$
a_{n}=\widetilde{b_{n}},
$$

where $a_{n}:=\int_{0}^{\pi} \rho(x) f(x) w_{1}\left(x, \lambda_{n}\right) d x$ and $\widetilde{b_{n}}:=\int_{0}^{\pi} \rho(x) g(x) \widetilde{w_{1}}\left(x, \lambda_{n}\right) d x$. Using the Parseval's equality (Adiloglu and Amirov (2013)), we calculate

$$
\|f\|_{L_{2}(0, \pi, \rho)}=\|g\|_{L_{2}(0, \pi, \rho)} .
$$

Now if we consider the operator

$$
A f(x)=f(x)+\int_{x}^{\pi} H(t, x) f(t) d t
$$

we have $A f=g$ and $\|A f\|_{L_{2}(0, \pi ; \rho)}=\|g\|_{L_{2}(0, \pi ; \rho)}$. Then by (38) we obtain

$$
\|A f\|_{L_{2}}=\|f\|_{L_{2}}
$$

for any $f(x) \in L_{2}(0, \pi)$. Then $A^{*}=A^{-1}$ is possible if and only if $H(t, x) \equiv 0$. Thus $w_{1}(x, \lambda)=\widetilde{w_{1}}(x, \lambda)$, i.e. $q(x)=\widetilde{q}(x)$ a.e. on $(0, \pi)$ and $h=\widetilde{h}, H=\widetilde{H}$. Theorem is proved. 
Let $q(x)=\alpha^{2} q(\alpha \pi-\alpha x)$ for $x>a$ and $h_{1}=\alpha h$. We now show that in this case the potential $q(x)$ and coefficient $h$ can be determined by the spectrum $\left\{\lambda_{n}^{2}\right\}_{n \geq 0}$ only.

Theorem 4. If $q(x)=\alpha^{2} q(\alpha \pi-\alpha x), x>a, h_{1}=\alpha h, \widetilde{q}(x)=\alpha^{2} \widetilde{q}(\alpha \pi-\alpha x), x>a, \widetilde{h_{1}}=\alpha \tilde{h}$ and $\lambda_{n}=\tilde{\lambda_{n}}$, $n \geq 0$ then $q(x)=\widetilde{q}(x)$ a.e. on $(0, \pi)$ and $h=\widetilde{h}$.

Proof. If $q(x)=\alpha^{2} q(\alpha \pi-\alpha x), x>a$ and $y(x)$ is a solution of the Eq. (1) for $0 \leq x \leq a$, then $y_{1}(x):=$ $y(\alpha \pi-\alpha x)$ is a solution of (1) for $x>a$.

Indeed, it is easy to check that

$$
-y_{1}^{\prime \prime}(x)=\rho(x) \lambda^{2} y_{1}(x), \text { i.e. }
$$

$y_{1}(x)$ is a solution for $x>a$. In particular, if we take the solution

$$
y(x)=-w_{1}(x, \lambda), \quad 0 \leq x \leq a
$$

then $y_{1}(x)=-w_{1}(\alpha \pi-\alpha x, \lambda),(x>a)$ is the solution of (1) satisfying the initial conditions $y_{1}(\pi)=$ $-w_{1}(0, \lambda)=-1, y_{1}^{\prime}(\pi)=\alpha w_{1}^{\prime}(0, \lambda)=\alpha h=h_{1}$.

Consequently, $w_{2}(x, \lambda) \equiv-w_{1}(\alpha \pi-\alpha x, \lambda)$. Now using $w_{2}\left(x, \lambda_{n}\right)=\beta_{n} w_{1}\left(x, \lambda_{n}\right), \beta_{n} \neq 0$ we have

$$
w_{2}\left(x, \lambda_{n}\right)=-\frac{1}{\beta_{n}} w_{1}\left(x, \lambda_{n}\right)
$$

which implies $\beta_{n}^{2}=1$. On the other hand $\beta_{n} w_{1}\left(\pi, \lambda_{n}\right)=-1$ and

$$
w_{1}\left(\pi, \lambda_{n}\right)=c_{0}\left(\pi, \lambda_{n}^{0}\right)+\frac{\xi_{n}(\pi)}{n},\left|\xi_{n}(\pi)\right| \leq C,(\text { see Adiloglu and Amirov 2013). }
$$

Consequently we have $\beta_{n}=(-1)^{n+1}$, hence from the formula $\beta_{n} \alpha_{n}=-\frac{\Delta\left(\lambda_{n}\right)}{2 \lambda_{n}}$ we obtain

$$
\alpha_{n}=\frac{(-1)^{n} \dot{\Delta}\left(\lambda_{n}\right)}{2 \lambda_{n}}
$$

Since $\lambda_{n}=\tilde{\lambda}_{n}$ we have $\alpha_{n}=\widetilde{\alpha_{n}}$. Then by the previous theorem $q(x)=\widetilde{q}(x)$ a.e. on $(0, \pi)$ and $h=\widetilde{h}$. Theorem is proved.

\section{BOUNDARY VALUE PROBLEMS $L^{0}$ AND $L_{1}$}

(i) Consider the boundary value problem $L_{1}=L_{1}(q(x), h)$ for equation (1) with the boundary conditions

$$
y^{\prime}(0)-h y(0)=0, y(\pi)=0 .
$$

The characteristic function of the problem $L_{1}$ is $d(\lambda)=w_{1}(\pi, \lambda)$ and the eigenvalues of $L_{1}$ are the squares of zeros of the equation $w_{1}(\pi, \lambda)=0$.

Note that as in the case of the problem $L$ we can prove that the eigenvalues $\left\{\mu_{n}^{2}\right\}$ of the problem $L_{1}$ are real and simple.

Since

$$
w_{1}(\pi, \lambda)=c_{0}(\pi, \lambda)+O\left(\frac{e^{\left|\operatorname{Im} \lambda \mu^{+}(\pi)\right|}}{|\lambda|}\right), \quad|\lambda| \rightarrow \infty
$$


we have for the eigenvalues $\left\{\mu_{n}^{2}\right\}$ the following asymptotic formula:

$$
\mu_{n}=\mu_{n}^{0}+\varepsilon_{n}
$$

where

$$
\mu_{n}^{0}=\frac{\pi}{\mu^{+}(\pi)}\left(n+\frac{1}{2}\right)+\theta_{n}, \quad\left(\sup _{n}\left|\theta_{n}\right|<+\infty\right)
$$

are the roots of the equation $c_{0}(\pi, \lambda)=0$ and $\varepsilon_{n}=o(1), n \rightarrow \infty$.

Further it is easy to show that (see Adiloglu and Amirov 2013 also) the solution $w_{1}(x, \lambda)$ satisfies the asymptotic relation

$$
w_{1}(x, \lambda)=c_{0}(x, \lambda)+h s_{0}(x, \lambda)+\int_{0}^{x} g(x, t, \lambda) c_{0}(t, \lambda) q(t) d t+O\left(\frac{e^{\left|\operatorname{Im} \lambda \mu^{+}(\pi)\right|}}{\lambda^{2}}\right), \lambda \rightarrow \infty
$$

where

$$
g(x, t, \lambda)=s_{0}(x, \lambda) c_{0}(t, \lambda)-c_{0}(x, \lambda) s_{0}(t, \lambda), 0 \leq x \leq \pi, 0 \leq t \leq \pi .
$$

Therefore we obtain

$$
\begin{gathered}
d(\lambda)=w_{1}(\pi, \lambda)=\alpha^{+} \cos \lambda \mu^{+}(\pi)+\alpha^{-} \cos \lambda \mu^{-}(\pi) \\
+b_{1} \alpha^{+} \frac{\sin \lambda \mu^{+}(\pi)}{\lambda}+b_{2} \alpha^{-} \frac{\sin \lambda \mu^{-}(\pi)}{\lambda}+\frac{k(\lambda)}{\lambda}
\end{gathered}
$$

where

$$
\begin{gathered}
b_{1}=h+\frac{1}{2} \int_{0}^{\pi} \frac{q(t)}{\sqrt{\rho(t)}} d t, \\
b_{2}=h+\frac{1}{2} \int_{0}^{\pi} \frac{\operatorname{sgn}(a-t)}{\sqrt{\rho(t)}} q(t) d t, \\
k(\lambda)=\int_{-\mu^{+}(\pi)}^{\mu^{+}(\pi)} Q(t) \sin \lambda t d t+O\left(\frac{e^{\left|\operatorname{Im} \lambda \mu^{+}(\pi)\right|}}{\lambda}\right), \lambda \rightarrow \infty
\end{gathered}
$$

with $Q(t) \in L_{2}\left(-\mu^{+}(\pi), \mu^{+}(\pi)\right)$.

Since $d\left(\mu_{n}\right)=0$, equations (40), and (41) imply that

$$
\begin{aligned}
& \left(\mu_{n}^{0}+\varepsilon_{n}\right)\left(\alpha \cos \left(\mu_{n}^{0}+\varepsilon_{n}\right) \mu^{+}(\pi)+\alpha^{-} \cos \left(\mu_{n}^{0}+\varepsilon_{n}\right) \mu^{-}(\pi)\right) \\
& +b_{1} \alpha^{+} \sin \left(\mu_{n}^{0}+\varepsilon_{n}\right) \mu^{+}(\pi)+b_{2} \alpha^{-} \sin \left(\mu_{n}^{0}+\varepsilon_{n}\right) \mu^{-}(\pi)+k_{n}=0
\end{aligned}
$$

and consequently

$$
\begin{gathered}
\mu_{n}^{0}\left[\alpha^{+} \mu^{+}(\pi) \sin \mu_{n}^{0} \mu^{+}(\pi)+\alpha^{-} \mu^{-}(\pi) \sin \mu_{n}^{0} \mu^{-}(\pi)\right] \varepsilon_{n} \\
=\mu^{+}(\pi) b_{1} \alpha^{+} \cos \mu_{n}^{0} \mu^{+}(\pi)+\mu^{-}(\pi) b_{2} \alpha^{-} \cos \mu_{n}^{0} \mu^{-}(\pi) \\
+b_{1} \alpha^{+} \sin \mu_{n}^{0} \mu^{+}(\pi)+b_{2} \alpha^{-} \sin \mu_{n}^{0} \mu^{-}(\pi)+k_{n},
\end{gathered}
$$

which implies

$$
\varepsilon_{n}=\frac{d_{n}}{\mu_{n}^{0}}+\frac{k_{n}}{n}
$$


where

$$
d_{n}=\frac{b_{1} \alpha^{+} \sin \mu_{n}^{0} \mu^{+}(\pi)+b_{2} \alpha^{-} \sin \mu_{n}^{0} \mu^{-}(\pi)}{\alpha^{+} \mu^{+}(\pi) \sin \mu_{n}^{0} \mu^{+}(\pi)+\alpha^{-} \mu^{-}(\pi) \sin \mu_{n}^{0} \mu^{-}(\pi)}
$$

$k_{n} \in l_{2}$. Hence

$$
\mu_{n}=\mu_{n}^{0}+\frac{d_{n}}{\mu_{n}^{0}}+\frac{k_{n}}{n} .
$$

Moreover if we define the normalized numbers $\left(\alpha_{n_{1}}\right)$ for the problem $L_{1}$ as

$$
\alpha_{n_{1}}=\int_{0}^{\pi} \rho(x) w_{1}^{2}\left(x, \mu_{n}\right) d x
$$

then we have

$$
\alpha_{n_{1}}=\alpha_{n_{1}}^{0}+\frac{t_{n_{1}}}{n}
$$

where

$$
\alpha_{n_{1}}^{0}=\int_{0}^{\pi} \rho(x) c_{0}^{2}\left(x, \mu_{n}\right) d x \text { and } t_{n_{1}} \in l_{2} .
$$

Since $d(\lambda)$ is an entire function of order one by the H'Adamard's theorem the function $d(\lambda)$ is uniquely determined up to a multiplicative constant $C$ by its zeros:

$$
d(\lambda)=C \prod_{n=0}^{\infty}\left(1-\frac{\lambda^{2}}{\mu_{n}^{2}}\right) .
$$

We also have

$$
c_{0}(\pi, \lambda)=\alpha^{+} \cos \lambda \mu^{+}(\pi)+\alpha^{-} \cos \lambda \mu^{-}(\pi)=\prod_{n=0}^{\infty}\left(1-\frac{\lambda^{2}}{\left(\mu_{n}^{0}\right)^{2}}\right)
$$

Since

$$
\lim _{|\lambda| \rightarrow \infty} \frac{d(\lambda)}{c_{0}(\pi, \lambda)}=1, \lim _{|\lambda| \rightarrow \infty} \prod_{n=0}^{\infty}\left(1+\frac{\mu_{n}^{2}-\left(\mu_{n}^{0}\right)^{2}}{\left(\mu_{n}^{0}\right)^{2}-\lambda^{2}}\right)=1
$$

we have

$$
C=\prod_{n=0}^{\infty} \frac{\mu_{n}^{2}}{\left(\mu_{n}^{0}\right)^{2}}
$$

therefore

$$
d(\lambda)=\prod_{n=0}^{\infty} \frac{\mu_{n}^{2}}{\left(\mu_{n}^{0}\right)^{2}}\left(1-\frac{\lambda^{2}}{\mu_{n}^{2}}\right)=\prod_{n=0}^{\infty}\left(\frac{\mu_{n}^{2}-\lambda^{2}}{\left(\mu_{n}^{0}\right)^{2}}\right) .
$$

We have proved the following two theorems:

Theorem 5. The boundary value problem $L_{1}=L_{1}(q(x), h)$ has a countable set of eigenvalues $\left\{\mu_{n}^{2}\right\}{ }_{n \geq 1}$ and for sufficiently large values of $n$, the asymptotic formula

$$
\mu_{n}=\mu_{n}^{0}+\frac{d_{n}}{\mu_{n}^{0}}+\frac{k_{n}}{n}
$$

are satisfied,where

$$
\begin{gathered}
\mu_{n}^{0}=\frac{\pi}{\mu^{+}(\pi)}\left(n+\frac{1}{2}\right)+\theta_{n},\left(\sup _{n}\left|\theta_{n}\right|<+\infty\right), \\
d_{n}=\frac{b_{1} \alpha^{+} \sin \mu_{n}^{0} \mu^{+}(\pi)+b_{2} \alpha^{-} \sin \mu_{n}^{0} \mu^{-}(\pi)}{\alpha^{+} \mu^{+}(\pi) \sin \mu_{n}^{0} \mu^{+}(\pi)+\alpha^{-} \mu^{-}(\pi) \sin \mu_{n}^{0} \mu^{-}(\pi)},
\end{gathered}
$$


is bounded and $k_{n} \in \ell_{2}$. Moreover, we have

$$
\alpha_{n_{1}}=\alpha_{n_{1}}^{0}+\frac{t_{n_{1}}}{n}
$$

for the normalized numbers

$$
\alpha_{n_{1}}=\int_{0}^{\pi} \rho(x) w_{1}^{2}\left(x, \mu_{n}\right) d x
$$

of the problem $L_{1}$, where

$$
\alpha_{n_{1}}^{0}=\int_{0}^{\pi} \rho(x) c_{0}^{2}\left(x, \mu_{n}^{0}\right) d x \text { and } t_{n_{1}} \in l_{2} .
$$

Theorem 6. The specification of the spectrum $\left\{\mu_{n}^{2}\right\}_{n \geq 0}$ uniquely determines the characteristic function $d(\lambda)=w_{1}(\pi, \lambda)$ by the formula

$$
d(\lambda)=\prod_{n=0}^{\infty}\left(\frac{\mu_{n}^{2}-\lambda^{2}}{\left(\mu_{n}^{0}\right)^{2}}\right) .
$$

(ii) Consider the boundary value problem $L^{0}=L^{0}\left(q(x), h_{1}\right)$ for equation (1) with the boundary condition

$$
y(0)=0, y^{\prime}(\pi)+h_{1} y(\pi)=0 .
$$

The eigenvalues $\left\{\left(\lambda_{n}^{0}\right)^{2}\right\}_{n \geq 0}$ of the problem $L^{0}$ are simple and coincide with the squares of zeros of the characteristic function

$$
\Delta^{0}(\lambda):=w_{2}(0, \lambda)=s^{\prime}(\pi, \lambda)+h_{1} s(\pi, \lambda)
$$

where $s(x, \lambda)$ is the solution of Eq.(1) with the initial conditions $s(0, \lambda)=0, s^{\prime}(0, \lambda)=1$.

From the results of Adiloglu and Amirov (2013), we have

$$
s(x, \lambda)=s_{0}(x, \lambda)+\int_{0}^{x} g(x, t, \lambda) q(t) s(t, \lambda) d t .
$$

Since

$$
\begin{gathered}
s(x, \lambda)=s_{0}(x, \lambda)+O\left(\frac{e^{\left|\operatorname{Im} \lambda \mu^{+}(x)\right|}}{\lambda^{2}}\right),|\lambda| \rightarrow \infty \\
s^{\prime}(x, \lambda)=s_{0}^{\prime}(x, \lambda)+\alpha \int_{0}^{a}\left[\alpha^{+} \cos \lambda\left(\mu^{+}(x)-t\right)-\alpha^{-} \cos \lambda\left(\mu^{-}(x)-t\right)\right] \frac{\sin \lambda t}{\lambda} q(t) d t \\
+\int_{a}^{x} \cos \lambda\left(\mu^{+}(x)-\mu^{+}(t)\right) q(t) d t s_{0}(x, \lambda)+O\left(\frac{e^{\left|\operatorname{Im} \lambda \mu^{+}(x)\right|}}{\lambda^{2}}\right),|\lambda| \rightarrow \infty,
\end{gathered}
$$

We have

$$
\begin{array}{r}
\Delta^{0}(\lambda)=\frac{\alpha \alpha^{+}}{2} \cos \lambda \mu^{+}(\pi)-\frac{\alpha \alpha^{-}}{2} \cos \lambda \mu^{-}(\pi) \\
+\alpha^{+} w_{+}^{0} \frac{\sin \lambda \mu^{+}(\pi)}{\lambda}+\alpha^{-} w_{-}^{0} \frac{\sin \lambda \mu^{-}(\pi)}{\lambda}+\frac{k^{0}(\lambda)}{\lambda}
\end{array}
$$

where

$$
w_{ \pm}^{0}=h_{1} \pm \frac{\alpha}{2} \int_{0}^{a} q(t) d t+\frac{1}{2} \int_{a}^{\pi} q(t) d t
$$




$$
\begin{gathered}
k^{0}(\lambda)=-\frac{\alpha \alpha^{+}}{2} \int_{0}^{\pi} \frac{q(t)}{\sqrt{\rho(t)}} \sin \lambda\left(\mu^{+}(\pi)-2 \mu^{+}(t)\right) d t+\frac{\alpha \alpha^{-}}{2} \int_{0}^{a} q(t) \sin \lambda\left(\mu^{-}(\pi)-2 t\right) d t \\
+\frac{\alpha \alpha^{-}}{2} \int_{0}^{\pi} q(t) \sin \lambda\left(2 \mu^{-}(t)-\mu^{-}(\pi)\right) d t+O\left(\frac{e^{\left|\operatorname{Im} \lambda \mu^{+}(\pi)\right|}}{|\lambda|}\right) .
\end{gathered}
$$

Therefore

$$
\begin{gathered}
\Delta^{0}(\lambda)=\frac{\alpha}{2}\left(\alpha^{+} \cos \lambda \mu^{+}(\pi)-\alpha^{-} \cos \lambda \mu^{-}(\pi)\right) \\
+\alpha^{+} w_{+}^{0} \frac{\sin \lambda \mu^{+}(\pi)}{\lambda}+\alpha^{-} w_{-}^{0} \frac{\sin \lambda \mu^{-}(\pi)}{\lambda}+\frac{k^{0}(\lambda)}{\lambda}
\end{gathered}
$$

From (58) we obtain the following expression for the roots $\left\{\lambda_{n}^{0}\right\}$ of the function $\Delta^{0}(\lambda)$ :

$$
\lambda_{n}^{0}=\gamma_{n}^{0}+\eta_{n}
$$

where

$$
\begin{gathered}
\gamma_{n}^{0}=\frac{\pi}{\mu^{+}(\pi)}\left(n+\frac{1}{2}\right)+\theta_{n}^{0} \\
\sup _{n}\left|\theta_{n}^{0}\right|<+\infty \text { and } \eta_{n}=o(1), n \rightarrow \infty .
\end{gathered}
$$

Since $\Delta^{0}\left(\lambda_{n}^{0}\right)=0$ some simple transformations lead to

$$
\begin{gathered}
\eta_{n}=\frac{d_{n}^{0}}{\gamma_{n}^{0}}+\frac{k_{n}^{0}}{n}, k_{n}^{0} \in l_{2}, \\
d_{n}^{0}=\frac{\alpha^{+} w_{+}^{0} \sin \mu^{+}(\pi) \gamma_{n}^{0}+\alpha^{-} w_{-}^{0} \sin \mu^{-}(\pi) \gamma_{n}^{0}}{\alpha \alpha^{+} \mu^{+}(\pi) \sin \mu^{+}(\pi) \gamma_{n}^{0}+\alpha \alpha^{-} \mu^{-}(\pi) \sin \mu^{-}(\pi) \gamma_{n}^{0}}
\end{gathered}
$$

where $\left(k_{n}\right) \in l_{2}$. Therefore

$$
\lambda_{n}^{0}=\gamma_{n}^{0}+\frac{d_{n}^{0}}{\gamma_{n}^{0}}+\frac{k_{n}^{0}}{n}, k_{n}^{0} \in l_{2}
$$

Moreover we can obtain that

$$
\Delta^{0}(\lambda)=\prod_{n=0}^{\infty} \frac{\left(\lambda_{n}^{0}\right)^{2}-\lambda^{2}}{\left(\gamma_{n}^{0}\right)^{2}}
$$

We can formulate the following theorem for the boundary- value problem $L^{0}$ :

Theorem 7. The boundary value problem $L^{0}=L^{0}\left(q(x), h_{1}\right)$ has a countable set of eigenvalues $\left\{\left(\lambda_{n}^{0}\right)^{2}\right\} n \geq 1$ and for sufficiently large values of $n$ the asymptotic formula (62) is satisfied. Moreover, the characteristic function

$$
\Delta^{0}(\lambda):=w_{2}(0, \lambda)=s^{\prime}(\pi, \lambda)+h_{1} s(\pi, \lambda)
$$

is uniquely determined by the spectrum $\left\{\left(\lambda_{n}^{0}\right)^{2}\right\}_{n \geq 0}$ via the formula (63).

Lemma 1. The following relation holds

$$
\lambda_{n}<\mu_{n}<\lambda_{n+1}, \quad n \geq 0,
$$

i.e. the eigenvalues of two boundary problems $L$ and $L_{1}$ are alternating. 
Proof. Consider the characteristic functions $\Delta(\lambda)$ and $d(\lambda)$ of the boundary value problems $L$ and $L_{1}$ respectively. Let $\lambda^{2}=z$. Then

$$
\Delta(\sqrt{z})=w_{1}^{\prime}(\pi, \sqrt{z})+h_{1} w(\pi, \sqrt{z}), d(\lambda)=w_{1}(\pi, \sqrt{z}) .
$$

Since

$$
\begin{aligned}
& (z-\mu) \int_{0}^{\pi} \rho(x) w_{1}(x, \sqrt{z}) w_{1}(x, \sqrt{\mu}) d x \\
& =(\Delta(\sqrt{\mu})-\Delta(\sqrt{z})) d(\sqrt{z})-(d(\sqrt{\mu})-d(\sqrt{z})) \Delta(\sqrt{z})
\end{aligned}
$$

for $\mu \rightarrow z$ we obtain

$$
\int_{0}^{\pi} \rho(x) w_{1}^{2}(x, \sqrt{z}) d x=\dot{\Delta}(\sqrt{z}) d(\sqrt{z})-\Delta(\sqrt{z}) \dot{d}(\sqrt{z})
$$

where

$$
\dot{\Delta}(\sqrt{z})=\frac{d}{d z} \Delta(\sqrt{z})
$$

and

$$
\dot{d}(\sqrt{z})=\frac{d}{d z} d(\sqrt{z})
$$

From (65) we have for $z=\lambda_{n}^{2}$

$$
\alpha_{n}=-\frac{\dot{\Delta}\left(\lambda_{n}\right) d\left(\lambda_{n}\right)}{2 \lambda_{n}}
$$

and

$$
\frac{1}{d^{2}(\sqrt{z})} \int_{0}^{\pi} \rho(x) w_{1}^{2}(x, \sqrt{z}) d x=-\frac{d}{d z}\left(\frac{\Delta(\sqrt{z})}{d(\sqrt{z})}\right),
$$

where $\lambda=(\sqrt{z})$ is real and $d(\sqrt{z}) \neq 0$.

Thus the function $\frac{\Delta(\sqrt{z})}{d(\sqrt{z})}$ is monotonically decreasing when $z \neq \mu_{n}^{2}, n \geq 0$ and

$$
\lim _{z \rightarrow\left(\mu_{n}^{ \pm 0}\right)^{2}} \Delta(\sqrt{z}) d^{-1}(\sqrt{z})= \pm \infty
$$

Then from asymptotic formulas for $\lambda_{n}$ and $\mu_{n}$, we arrive at (64).

\section{ACKNOWLEDGMENTS}

We thank the referees for their encouraging remarks and insightful comments. Also, this work is supported by the Scientific and Technological Research Council of Turkey (TÜBİTAK) with Project 113F366.

\section{REFERENCES}

ADILOGLU NA AND AMIROV RKH. 2013. On the boundary value problem for the Sturm-Liouville equation with the discontinuous coefficient. Math Methods Appl Sci 36: 1685-1700.

AKHMEDOVA EN AND HUSEYNOV HM. 2010. On inverse problem for Sturm-Liouville operator with discontinuous coefficients. Proc of Saratov University, New ser. Ser Math Mech and Inf 10: 3-9.

AMIROV RKH. 2006. On Sturm-Liouville operators with discontinuity conditions inside an interval. J Math Analysis Appl 317:

163-176.

ANDERSSON L. 1988. Inverse eigenvalue problems for a Sturm-Liouville equation in impedance form. Inverse Probl 4: $929-971$. 
CARLSON R. 1994. An inverse spectral problem for Sturm-Liouville operators with discontinuous coefficients. Proc Amer Math Soc 120: 475-484.

FREILING G AND YURKO V. 2001. Inverse Sturm-Liouville problems and their applications, Nova Science Publishers, Inc., Huntington: NY, 305 p.

GASYMOV MG. 1977. The direct and inverse problem of spectral analysis for a class of equations with a discontinuous coefficient. Non-Classical Methods in Geophysics, 37-44, Nauka, Novosibirsk, Russia.

GUSEINOV IM AND PASHAEV RT. 2002. On an inverse problem for a second-order differential equation. Russian Math Surveys 57: 597-598.

HALD O. 1980. Inverse eigenvalue problems for the mantle. Geophys J R Astron Soc 62: 41-48.

HALD O. 1984. Discontinuous inverse eigenvalue problems. Commun Pure Appl Math 37: 539-577.

HRYNIV RO AND MYKYTYUK YAV. 2003. Inverse spectral problems for Sturm-Liouville operators with singular potentials. Inverse Probl 19: 665-684.

HRYNIV RO AND MYKYTYUK YAV. 2004. Half-inverse spectral problems for Sturm-Liouville operators with singular potentials. Inverse Probl 20: 1423-1444.

KRUEGER R. 1982. Inverse problems for nonabsorbing media with discontinuous material properties. J Math Phys 23: $396-404$.

LEVITAN BM. 1987. Inverse Surm-Liouville problems, VNU Sci. Press, Utrecht, 240 p.

LEVITAN BM AND GASYMOV MG. 1964. Determination of a differential equation by two spectra. Uspehi Mat Nauk 19: 3-63.

MAMEDOV KHR. 2006. Uniqueness of the solution of the inverse problem of scattering theory for Sturm-Liouville operator with discontinuous coefficient. Proc Inst Math Mech Natl Acad Sci Azerb 24: 163-172.

MAMEDOV KHR. 2010. On an inverse scattering problem for a discontinuous Sturm-Liouville equation with a spectral parameter in the boundary condition. Bound Value Probl ID 171967, 17 p.

MAMEDOV KHR AND PALAMUT N. 2009. On a direct problem of scattering theory for a class of Sturm-Liouville operator with discontinuous coefficient. Proc Jangjeon Math Soc 12: 243-251.

MARCHENKO VA. 2011. Sturm-Liouville operators and applications, AMS Chelsea Publishing Volume 373, 393 p.

WILLIS C. 1984. Inverse problems for torsional modes. Geophys J R Astron Soc 78: 847-853.

YURKO VA. 2000. On boundary value problems with discontinuity conditions inside an interval. Differ Equ 36: $1266-1269$. 